\title{
REGIONAL GROSS DOMESTIC PRODUCT PREDICTION USING TWITTER DEEP LEARNING REPRESENTATIONS
}

\author{
Javier Ortega-Bastida ${ }^{1}$, Antonio-Javier Gallego ${ }^{1}$, Juan Ramón Rico-Juan ${ }^{1}$ and Pedro Albarrán ${ }^{2}$ \\ ${ }^{1}$ Department of Software and Computing Systems, University of Alicante, Spain \\ ${ }^{2}$ Fundamentos del Análisis Económico (FAE), University of Alicante, Spain
}

\begin{abstract}
This work presents a method to predict the regional Gross Domestic Product (GDP) using the textual information stored in tweets. In particular, we propose the use of a hybrid autoencoder to predict the GDP of the Valencian Community (Spain) using the tweets written by the most influential economists, politicians, newspapers, and institutions in the region. The proposed method uses an autoencoder that is trained to simultaneously minimize the reconstruction error of the textual information of tweets along with their corresponding GDP value. In this way, we ensure that the latent space of the autoencoder represents the important characteristics of tweets that relate them to the GDP. This system allows to obtain a representation of new tweets that is related to a GDP prediction and also to analyze them to create clusters that represent the agreement or disagreement of the opinions. The results show that the proposed approach captures the GDP growth trend with a small margin of error. This method was also compared with other methods from the state of the art showing a better result in prediction accuracy.
\end{abstract}

\section{KEYWORDS}

Machine Learning, Neural Networks, Knowledge-Based Economy

\section{INTRODUCTION}

Since the emergence of Machine Learning (ML) and Deep Learning (DL), the study of problems in fields where sequential variables are important, such as in Economics, has led to significant progress over traditional statistical models, both in forecasting precision and runtime as it has been proven in Mullainathan \& Spiess (2017). As can be seen in LeCun et al. (2015), DL has made a breakthrough in the ability to learn suitable features from unstructured or sequential data, which makes the use of this type of technique especially interesting to improve the results obtained with the traditional approaches used in Economics.

Recent studies (Llorente et al. (2015) and Bokányi et al. (2017)) show the convenience of taking into account social media as a reflection of economic activity using ML techniques. Twitter is a huge database that captures the constant activity of users and, therefore, it can be used as an additional source of information in economic studies. An Interesting application is the extraction of relevant information from messages to apply sentiment analysis techniques like in Joshi \& Deshpande (2018). This Area of Natural Language Processing (NLP) allows estimating the opinion of people and, therefore, to extract trends, which is of special interest to complement solutions based on econometrics. For example, in Pagolu et al. (2016), DL techniques are used to find correlations between economic magnitudes and opinions expressed on Twitter. Besides, these results compete in quality with traditional approaches and have the advantage of being able to calculate them immediately.

Official statistics for relevant macroeconomic aggregates such as Gross Domestic Product (GDP) or unemployment rate are mainly focused on the annual magnitude and evolution for the whole country. Since policy making often requires information at lower frequencies (quarterly or monthly basis) and also for lower geographical units (regions or cities), the National Offices of Statistics compute some of these economic indicators using their interpolation methodologies (which are not always public and clear). In Spain, GDP is publicly available on an annual and quarterly basis, but regional GDP is only computed annually and no official figure is provided for provinces or cities. Therefore, new approaches that fill-in this gap with some (unofficial) magnitude should be useful. In the literature, there are several proposals for calculating GDP 
using ML techniques. For example, Zukime \& Junoh (2004) and Richardson et al. (2018) have shown the benefit of using different ML techniques -such as Neural Networks- for this purpose. They predict GDP using several macroeconomic aggregates, obtaining better and more accurate results than with traditional statistical models. Indaco (2019) has shown that Twitter is a perfect social network to estimate economic activity and GDP variation using the opinion of its users.

This paper proposes a method to predict the regional GDP of the Valencian Community (Spain: http://www.iaes.es/contabilidadregionaltrimestral.html) using only the Twitter Activity of the most influential economists, politicians, newspapers and institutions of that geographical area. This method uses a hybrid autoencoder that is trained to simultaneously minimize the reconstruction error of the textual information of tweets along with their corresponding GDP value. It allows us to synthesize the most important information and to provide a signal about the evolution of the GDP. Thus, the main objective of this work is to study to what extent Twitter interactions contain relevant information about economic activity. In particular, we predict GDP growth using only key information from this social network and we also analyze how accurately this method can reproduce the economic growth trends.

The analysis of the latent space learned by the autoencoder allows us to detect both the opinions that reach a greater consensus and those that move further away from the general opinion. We can also know those topics relevant to the economic evolution of that period. The proposed method analyzes this representation of the latent space to remove atypical opinions before calculating the final prediction. Results show that the proposed method can capture the trend of regional GDP growth. The method is compared with other approaches, including three state-of-the-art methods, showing better accuracy in all cases.

The remainder of this paper is organized as follows. Section 2 describes the methodology. Section 3 depicts the dataset used. Section 4 details the evaluation results. Section 5 addresses the conclusions.

\section{METHODOLOGY}

Figure 1 shows the proposed approach for GDP prediction. First, tweets are preprocessed and codified using a pre-trained embedding library. An autoencoder is then used to learn an intermediate representation that is associated with both tweets and GDP. This autoencoder uses a hybrid architecture in which not only the text is reconstructed but also the corresponding GDP. In this way, we ensure that the latent space of the autoencoder learns a representation of tweets that is related to the GDP. The latent space (or neural codes) of tweets is then filtered and, finally, a regressor method is used to predict the GDP.
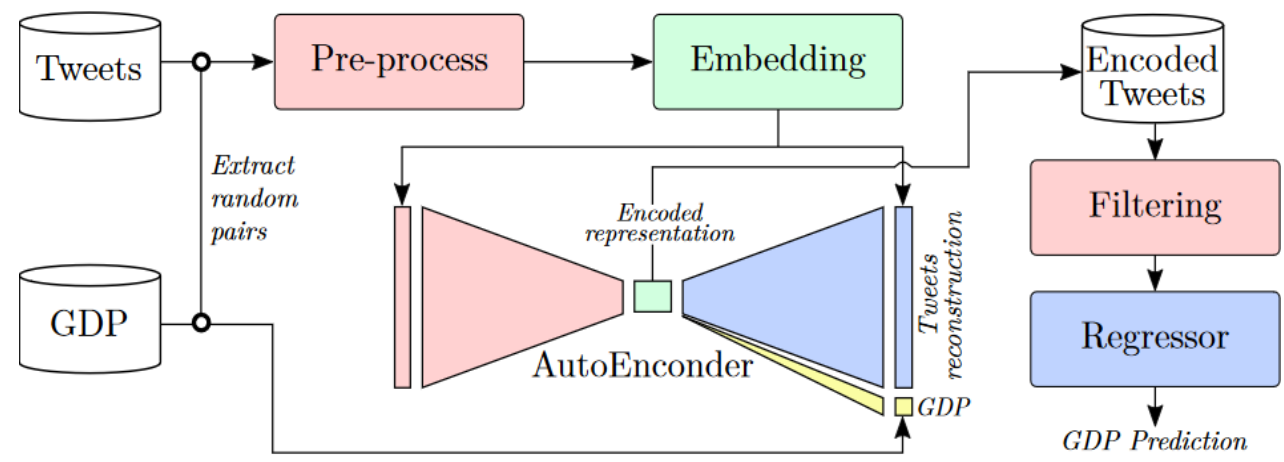

Figure 1. Overall architecture of the proposed method

\subsection{Tweets Preprocessing}

As is described in Kumar \& Sebastian (2012) (Section "Preprocessing of Tweets"), we preprocess tweets by following the steps: 1) Remove links, special characters and punctuation, 2) Delete stop words, 3) Lowercase the text, 4) Codify the text using a pretrained embedding model and 5) Normalize the codified tweets. 
Since tweets are text strings where the meaning of the words is conditioned by the context of the sentence, alternatives such as one-hot encoding are less convenient than other approaches such as Word2Vec (Mikolov et al., 2013) or Sense2Vec (Trask et al., 2015). The results shown in Ortega-Bastida (2019) comparing the classification using textual data demonstrate that the approach using neural coding models exceeds the one-hot encoding.

For the fifth step of the pre-processing, we use Sense2Vec (using the spaCy library implementation: https://spacy.io/models/es) as a sentence embedding method, which improves the results obtained by other word embedding models such as Word2 Vec that do not preserve the semantic meaning of the words within the context of the sentence. The spaCy library includes two pre-trained models for the Spanish language. Initially, this method was tested using both models with our dataset, obtaining a result similar to that reported by Trask et al. (2015). As a result of this encoding, each sentence is codified into a feature vector with 50 dimensions. The values of this vector are then normalized using $l 2$ and obtaining a better result when applying this normalization. These results agree with those also reported in Gallego et al. (2018).

\subsection{Hybrid Autoencoder}

The normalized embedding obtained in the previous step is supplied to a special type of autoencoder (see Figure 1) that receives one input (the embedding) and returns two outputs (the embedding reconstruction and the corresponding GDP). Therefore, this hybrid autoencoder is trained to minimize simultaneously the reconstruction error of the textual information of tweets along with the corresponding GDP value. For The training of this network, a loss function $(L r)$ is proposed, which combines the result of the reconstruction of the tweets and the prediction of GDP, as can be seen in the following equation:

$$
L r=\lambda \sum_{i=1}^{n}\left(t_{i}-\widehat{t_{l}}\right)^{2}+(1-\lambda) \sum_{i=1}^{n}\left(g_{i}-\widehat{g}_{l}\right)^{2}
$$

where $\widehat{t}_{l}$ is the tweet prediction, $\widehat{g}_{l}$ the GDP prediction, $t_{i}$ and $g_{i}$ the actual tweet and GDP values, respectively, $n$ the number of samples, and $\lambda$ allows us to adjust the weight assigned to each part of the network reconstruction, that is, give more weight to the reconstruction of tweets or more weight to GDP. The loss weight $\lambda$ was evaluated to determine its optimal value (see Section 4.1), eventually selecting a weight of 0.9 for GDP and 0.1 for the tweets reconstruction. The specific topology of an autoencoder can be very varied. In this paper, we study three possibilities: Vanilla autoencoder (a neural network with only fully connected layers), a CNN-1D (a Convolutional Neural Network that uses one-dimensional convolution), and an LSTM (a neural network composed of Long Short-Term Memory layers described by Shi et al. (2015)).

Table 1. Topologies considered. $F C(n)$ : a fully-connected layer of $n$ neurons. Conv1D $(f, k)$ : a one-dimensional convolution layer with $f$ filters and a kernel of size $k$. $L S T M(f)$ : a Long Short-Term Memory layer with $f$ filters

\begin{tabular}{|c|c|c|c|c|c|}
\hline Model & Layer 1 & Layer 2 & Layer 3 & Layer 4 & Output \\
\hline Vanilla & $\mathrm{FC}(64)$ & $\mathrm{FC}(32)$ & $\mathrm{FC}(32)$ & $\mathrm{FC}(64)$ & $\mathrm{FC}(50), \mathrm{FC}(1)$ \\
\hline CNN-1D & Conv1D $(64,3)$ & Conv1D $(32,3)$ & Conv1D $(32,3)$ & Conv1D(64,3) & $\mathrm{FC}(50), \mathrm{FC}(1)$ \\
\hline LSTM & $\operatorname{LSTM}(64)$ & $\operatorname{LSTM}(32)$ & $\operatorname{LSTM}(32)$ & $\operatorname{LSTM}(64)$ & $\mathrm{FC}(50), \mathrm{FC}(1)$ \\
\hline
\end{tabular}

Table 1 shows the implementation details of these three models. For all of them, two layers of encoding and two layers of decoding are considered, they receive a 50-dimensional vector as input and return two outputs, one with 50 dimensions (representing the tweets reconstruction), and an additional output with 1 dimension (with the GDP prediction, which is connected to the latent space of the network). ReLU was used in all layers as activation function except for the output layers, where a linear activation function was used for both outputs. The learning of the network weights was performed during 100 epochs using stochastic gradient descent and using Adam optimization with a mini-batch size of 32 samples. 


\subsection{Prediction Step}

In this last stage, the training set is forwarded through the autoencoder to store its intermediate representation. The resulting set of encoded data is then filtered to remove possible outliers and finally used to train a ML regressor. The filtering process allows us to remove atypical opinions, selecting only those that reach a greater consensus. For this, we use the Local Outlier Factor method explained in Breunig et al. (2000), with a margin of 35 neighbors and contamination of 0.5 . This method searches for anomalous data points by measuring the local deviation of a given data point to its neighbors.

For the GDP prediction, we propose the use of $\boldsymbol{\varepsilon}$-Support Vector Regression ( $\boldsymbol{\varepsilon}$-SVR) detailed by Vapnik (1998), which is trained using the encoded training set previously filtered. Different alternatives were evaluated, including the use of a simple linear regression, the output of the autoencoder with and without filtering, as well as the use of a Random Forests Regressor (RFr), explained by Breiman (2001), instead of $\varepsilon$-SVR. The results of this comparison can be seen in the experimentation section, in which it was determined that $\boldsymbol{\varepsilon}$-SVR obtained the best result.

To predict the GDP of a new quarter during the inference stage, the following steps are taken: 1) tweets are preprocessed, encoded and normalized as explained in Section 2.1,2) the resulting embedding is forwarded through the trained autoencoder and its intermediate representation is stored, and 3) $\boldsymbol{\varepsilon}$-SVR is used to predict the GDP. This will return a prediction for each tweet of the new set, to obtain the final prediction from this result we first filter the set obtained and then calculate the average using only those tweets whose opinion shows a greater consensus.

\section{DATASET}

For the evaluation of the proposed method, we used a dataset with a total of 30,000 tweets. It includes 1,500 tweets per quarter in the period 2012 to 2016. Tweets were obtained from the most influential and relevant accounts of the Valencian Community (Spain) in the economic field, including economists, radio channels, regional televisions, political leaders, communiques from town halls and official institutions of the regional government, finance counseling, economic newspapers, and regional branches of national newspapers. Table 2 shows some examples of the accounts chosen along with their type and description.

Table 2. Some examples of the Twitter accounts used in the dataset

\begin{tabular}{|c|c|c|}
\hline Type & Definition & Accounts \\
\hline Newspaper & $\begin{array}{l}\text { Local newspapers or regional sections } \\
\text { of national newspapers }\end{array}$ & $\begin{array}{l}\text { @lasprovincias, @larazoncv, } \\
\text { @elmundocv, @elpaisvalencia }\end{array}$ \\
\hline Economic Experts & $\begin{array}{l}\text { Newspapers and Groups specialized in } \\
\text { economics }\end{array}$ & $\begin{array}{l}\text { @elEconomistaCV,@Economia3,@In } \\
\text { stitutoINECA }\end{array}$ \\
\hline Journalists \& economists & $\begin{array}{l}\text { Personal accounts of economists, } \\
\text { professors or journalists }\end{array}$ & $\begin{array}{l}\text { @JMaudos,@DaValero,@Fpiera,@nd } \\
\text { elatowe }\end{array}$ \\
\hline Political parties & $\begin{array}{l}\text { Political party accounts and personal } \\
\text { accounts of politicians }\end{array}$ & $\begin{array}{l}@ \text { PSOEValencia,@ppcv,@ximopuig, } \\
@ \text { RubenIBB }\end{array}$ \\
\hline Radio \& television & $\begin{array}{l}\text { Regional Television and Radio } \\
\text { accounts }\end{array}$ & @InformacionTV, @LevanteTV \\
\hline $\begin{array}{l}\text { Public administration government } \\
\text { sectors }\end{array}$ & $\begin{array}{l}\text { Accounts from regional government } \\
\text { and administration }\end{array}$ & $\begin{array}{l}@ \text { GVAhisenda,@GVAivace,@GVAe } \\
\text { conomia }\end{array}$ \\
\hline
\end{tabular}

In addition to selecting tweets only from the accounts indicated above, we also filter them using a series of keywords related to GDP, such as: gdp, economic growth, growth forecasts, public debt, family debt, job creation, service sector, exports, imports, investment, tourism income, light price, inflation, confidence indicator, among others. In this way, we created a dataset with opinions not only of people related to the economy but of opinions that were related to GDP. Since the objective is to predict the GDP of the current quarter using the opinions of the quarter itself (note that the regional GDP is only officially published annually), tweets were labeled with the difference between the GDP of the quarter in which they were written with respect to the GDP of the previous quarter with the intention of knowing if they reflect an increase or decrease in GDP. This difference was calculated as the percentage and normalized in the range [-1,1]. In all experiments we used 5-fold cross-validation, using one year for test and the rest for training. Therefore, the autoencoder was trained and evaluated 5 times using these sets, after which the average result was calculated. 


\section{EXPERIMENTS}

\subsection{Autoencoder Results}

First, we evaluate the influence of the $\lambda$ hyper-parameter (see Equation 1) on the result obtained. For this, the Vanilla network (see Section 2.2) is trained by varying the weight $\lambda$ from 0 to 1 with increments of 0.1 . Figure 2 shows the result of this experiment in terms of Mean Square Error (MSE). As can be seen, the best result is obtained when $\lambda=0.1$ for the GDP output.

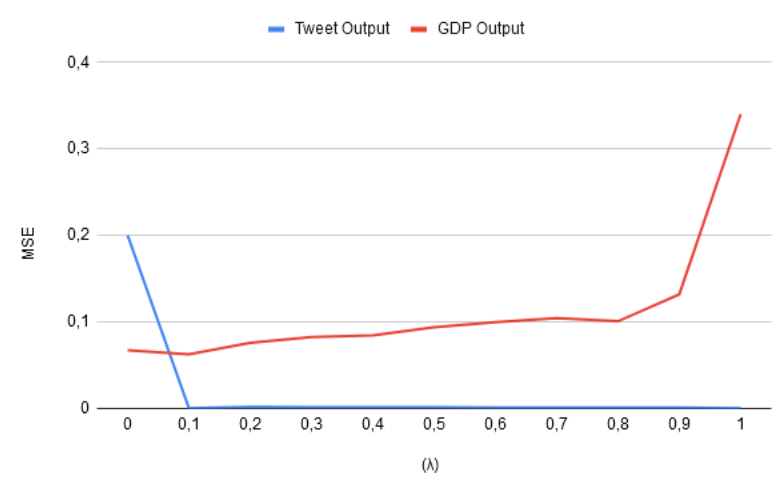

Figure 2. Result obtained (MSE) by varying the weight $(\lambda)$ with the contribution of each output of the hybrid network

Table 3 shows a comparison of the result obtained with the topologies considered using the best $\lambda$ value found in the previous experiment. The best result for tweet reconstruction and GDP prediction is obtained using the LSTM network. Therefore, we will use this topology to perform the following experiments.

Table 3. Comparison of the result (MSE) obtained with each network topology

\begin{tabular}{lccc}
\hline & Vanilla & CNN-1D & LSTM \\
\hline Tweet & 0.0019 & 0.0014 & $\mathbf{0 . 0 0 0 2}$ \\
GDP & 0.0626 & 0.0623 & $\mathbf{0 . 0 6 2 1}$ \\
\hline
\end{tabular}

\subsection{GDP Prediction}

Once the network is trained, we proceed to evaluate the next part of the method which calculates the GDP prediction using a regressor ( $\boldsymbol{\varepsilon}$-SVR) and filtering the atypical opinions (see Section 2.3). To evaluate this result, we use the Mean Absolute Error (MAE) metric, since it is a more intuitive metric to analyze the actual result. It must be taken into account that in this section we evaluate the final GDP prediction, while in the previous section we evaluated the reconstruction error of each tweet individually. Table 4 shows the result obtained with the proposed method (AE $+\mathrm{NC}$ Filtered $+\boldsymbol{\varepsilon}$-SVR) for each of the years evaluated. This table also shows the result obtained with five other methods: two basic baselines and the results obtained in each of the steps of the proposed method, that is, using only the autoencoder, adding the filtering process, and also comparing with the use of a different regressor. A better description of the compared methods follows:

1) Baseline: It assumes that GDP does not vary from quarter to quarter. That is, this method predicts that the GDP of the current quarter will be equal to the actual GDP of the previous quarter.

2) Linear regression: The prediction is calculated using the GDP value of the previous four quarters.

3) AutoEncoder (AE): The GDP of all tweets of the evaluated quarter is predicted using the autoencoder and the average of these predictions is then calculated.

4) $\mathrm{AE}+\mathrm{NC}$ Filtered: This approach does the same as the previous one but also applying a filtering process before calculating the average prediction.

5) $\mathrm{AE}+\mathrm{NC}$ Filtered $+R F r$ : This approach performs the same steps as the proposed method (AE + NC Filtered $+\varepsilon-S V R$ ) but changing the regressor by a Random Forest Regressor $(R F r)$. 
Table 4. Comparison of the result obtained (MAE) using the proposed method with five other approaches

\begin{tabular}{lllllll}
\hline Method & $\mathbf{2 0 1 2}$ & $\mathbf{2 0 1 3}$ & $\mathbf{2 0 1 4}$ & $\mathbf{2 0 1 5}$ & $\mathbf{2 0 1 6}$ & Avg. \\
\hline Baseline & 1.25 & 1.06 & 1.30 & 1.14 & 1.22 & 1.19 \\
Linear Regression & 0.51 & 0.78 & 0.94 & 0.55 & 0.58 & 0.67 \\
AutoEncoder (AE) & 0.41 & 0.37 & 0.48 & 0.38 & 0.42 & 0.41 \\
AE + NC Filtered & 0.38 & 0.34 & 0.44 & 0.35 & 0.39 & 0.38 \\
AE + NC Filtered + RFr & 0.28 & 0.25 & 0.32 & 0.25 & 0.29 & 0.27 \\
Our Approach (AE + NC Filtered + $\boldsymbol{\varepsilon}$-SVR) & $\mathbf{0 . 2 5}$ & $\mathbf{0 . 2 3}$ & $\mathbf{0 . 3 0}$ & $\mathbf{0 . 2 4}$ & $\mathbf{0 . 2 7}$ & $\mathbf{0 . 2 5}$ \\
\hline
\end{tabular}

The proposed method $(\mathrm{AE}+\mathrm{NC}$ Filtered $+\boldsymbol{\varepsilon}$-SVR) obtains the best result for all years, with the AE $+\mathrm{NC}$ Filtered + RFr approach being the second-best result. It can be seen how the proposed method significantly improves simpler approaches such as the baseline or the linear regression, improving them by 0.94 and 0.42 .

Figure 3a shows a graph with the GDP prediction for each quarter, this result is compared with the actual GDP value. It can be seen how our proposed method closely tracks GDP evolution over time. In particular, our prediction can detect the seasonal up-and-downs of the regional GDP and its general trend using only tweets written in that quarter. This fact is indicated in the figure by the dotted line, which highlights the differences between the seasonal values (only for the rising stations). In addition to capturing the seasonal trend, it also detects the differences between these rises and falls, that is, the irregular component of this trend which indicates whether the quarter is going to increase more or less compared to the previous quarter.

To better analyze the features learned by the network, we use the t-Distributed Stochastic Neighbor Embedding (t-SNE) projection proposed by van der Maaten \& Hinton (2008) to visualize the feature distribution learned by the latent space of the autoencoder. Figure $3 \mathrm{~b}$ shows two examples for two random quarters. Each representation is calculated separately, so it should only show a cluster, however, there is a diversity of opinions for the same GDP in which some of them move away from the rest or create small clusters. As it was shown, the result obtained improved by removing these outliers.
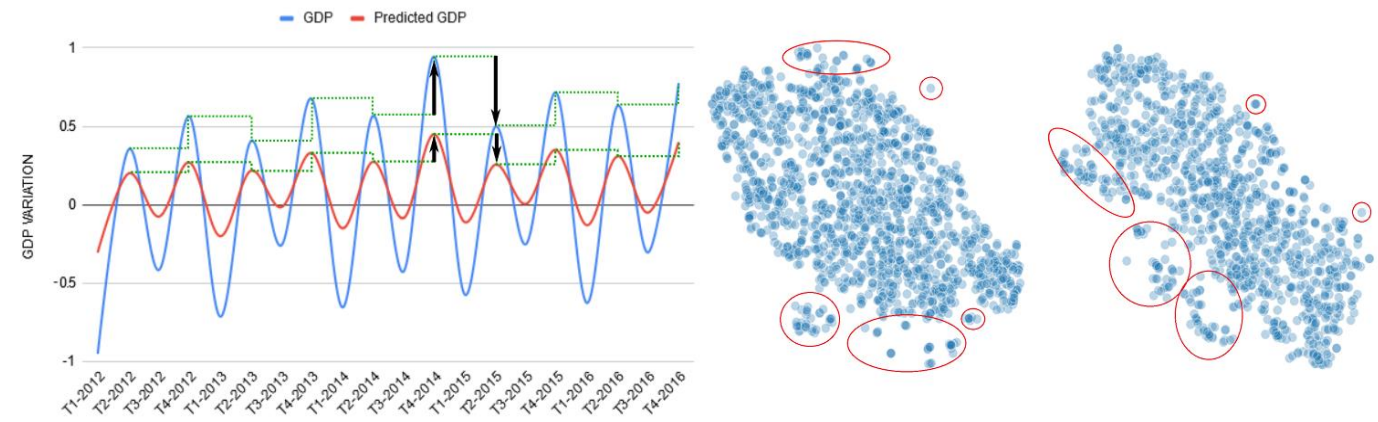

Figure 3. (a) Comparison of the GDP prediction made by the proposed method with the actual GDP value. (b) Feature distribution learned by the latent space of the autoencoder for two random quarters using t-SNE

\subsection{Comparison}

In this section, the proposed method is compared with the following three state-of-the-art methods:

- Indaco (2019) estimates the GDP of different countries around the world making a correlation between the country and the volume of the 270 million geolocated images shared on Twitter. This method uses a feature vector with some characteristics of the countries like the national population.

- Richardson et al. (2018) evaluate multiple approaches to predict the GDP by managing several economic indicators and different ML models like Autoregressive Model, SVM or feedforward NN.

- Zukime \& Junoh (2004) apply a Fully Connected NN trained to predict the GDP using economic indicators. The Network consists of 4 input units (corresponding to the indicators chosen), one hidden layer and one output.

Table 5 shows the result of this comparison using the MAE and RMSE metrics. For Indaco (2019) and Richardson et al. (2018), we selected the methods and configuration of parameters that according to the authors achieved the best accuracy. As can be seen, the proposed method improves the other results. 
Table 5. Comparison with other methods. The symbol "-" indicates that these results are not provided

\begin{tabular}{lcc}
\hline Method & RMSE & MAE \\
\hline Indaco (2019) & 0.53 & - \\
Richardson et al. (2018) & 0.42 & - \\
Zukime \& Junoh (2004) & 0.57 & 0.33 \\
Our approach & $\mathbf{0 . 3 6}$ & $\mathbf{0 . 2 5}$ \\
\hline
\end{tabular}

\subsection{Discussion}

One additional advantage of our method relies on the possibility of an ex-post analysis of the filtered tweets to obtain relevant insights. As a by-product of our procedure, we can identify those tweets that are more (and less) influential in the sense of achieving a closer (or further) prediction to the GDP. By analyzing the set of most influential and least influential tweets we can know those topics relevant in the economic evolution of each year. Table 6 reports a few of the most and least influential tweets for every year in our study.

Table 6. Some examples of the most and least influential tweets

\begin{tabular}{|c|c|c|}
\hline Years & Most Influential Tweets & Least Influential Tweets \\
\hline 2012 & Spain, one step away from the recession after its GDP fell $0.3 \%$. & The ECB doesn't give the guarantees that Rajoy asks for. \\
\hline 2013 & GDP moderates its contraction to $0.5 \%$ in the first quarter. & s rates at the historical low of $0.75 \%$ \\
\hline 2014 & Advances that GDP will grow $1 \%$ in 2014 and $1.5 \%$ in 2015 & Consell will double the investment in 2015 with a $10 \mathrm{M}$ item. \\
\hline 2015 & The exports of the CV grew $6 \%$ in January-November. & The ECB keeps rates at the historical low of $0.05 \%$. \\
\hline 2016 & $\begin{array}{l}\text { Cevisama } 2016 \text { confirms the recovery of the ceramic sector, which } \\
\text { leaves fear behind. }\end{array}$ & $\begin{array}{l}\text { Puig asks for another meeting with Montoro for the FLA and } \\
\text { the Brussels fine for the deficit. }\end{array}$ \\
\hline
\end{tabular}

In the analysis of the most influential tweets, we can follow the path of the Spanish economy. In 2012, these tweets contain negative terms such as recession, GDP fall, investment fall, etc. Although negative news still prevails in 2013, some positive tones arise: housing prices keep falling and the administration's debt increasing. The beginning of the good macroeconomic news comes in 2014: GDP is expected to grow, debts with suppliers begin to stabilize and the risk premium falls. These dynamics continue in 2015 with a mostly positive overall balance: exports are going well and the payment time to suppliers is reduced. In 2016, GDP recovery is finally accompanied by employment creation, but some areas continue a negative trend, damaging the average balance. In the set of tweets with worse forecasting precision, we find tweets that contain generic information from politicians or those who analyze the entire country and do not provide specific information about the region. We also noticed that tweets commenting on information about previous quarters or years do not contribute to our prediction either.

\section{CONCLUSIONS AND FUTURE WORK}

In this work, we propose a method to predict the regional GDP of the Valencian Community (Spain) using the Twitter activity of the most influential economists, politicians, newspapers, and institutions of that geographical area. For this, a hybrid autoencoder is trained to simultaneously reconstruct the textual information of tweets along with their corresponding GDP value. $\boldsymbol{\varepsilon}$-SVR regressor is then trained to predict GDP using the latent space learned by the autoencoder. The analysis of this encoded representation allows us to detect both the opinions that reach a greater consensus and those that move further away from the general opinion. The final prediction is calculated by averaging the GDP prediction of the tweets whose opinion shows a greater consensus. Different network topologies and hyper-parameters were evaluated to obtain the best configuration. Results show that the proposed method can capture the trend of regional GDP growth. The method was compared with other approaches, including three state-of-the-art methods, showing better accuracy in all cases. This method has been able to extract information from Twitter using exclusively the activity from a set of its most influential users. Such information has been proved to accurately predict on its own how GDP evolves. The work shows that social networks can be used to provide a fairly close reflection of economic prospects in real-time and for geographical units where official statistics are not available. 
As future work, it is intended to improve the accuracy of the proposed method by adding economic variables collected from the different territorial levels that are not used in the GDP national calculation (such as household consumption). Besides, we also want to extend the experiments to other regions of Spain.

\section{ACKNOWLEDGEMENT}

This work was supported by the Pattern Recognition and Artificial Intelligence Group (PRAIg) from the University of Alicante, Spain.

\section{REFERENCES}

Bokányi, E., Lábszki, Z. and Vattay, G., "Prediction of employment and unemployment rates from twitter daily rhythms in the us," EPJ Data Science, vol. 6, no. 1, Jul 2017.

Breiman, L., "Random forests," Machine Learning, vol. 45, no. 1, Oct 2001, pp. 5-32.

Breunig, M. M., Kriegel, H.-P., Ng, R. T. and Sander, J., "Lof: Identifying density-based local outliers,” SIGMOD Rec., vol. 29, no. 2, May 2000, p. 93-104.

Gallego, A.-J., Pertusa, A. and Calvo-Zaragoza, J., “Improving convolutional neural networks' accuracy in noisy environments using k-nearest neighbors," Applied Sciences, vol. 8, no. 11, 2018.

Indaco, A., "From Twitter to GDP: Estimating Economic Activity from Social Media," University Library of Munich, Germany, MPRA Paper 95885, Mar. 2019.

Joshi, S. and Deshpande, D., "Twitter sentiment analysis system," International Journal of Computer Applications, vol. 180 , no. 47 , Jun 2018 , p. 35-39.

Kumar, A. and Sebastian, T., "Sentiment analysis on twitter," International Journal of Computer Science Issues, vol. 9 , 07 2012, pp. 372-378.

LeCun, Y., Bengio, Y. and Hinton, G., "Deep learning,” Nature, vol. 521, no. 7553, 2015, pp. 436-444.

Llorente, A., Garcia-Herranz, M., Cebrian, M. and Moro, E., "Social media fingerprints of unemployment," PLOS ONE, vol. 10, no. 5, May 2015, p. e 0128692.

Mikolov, T., Sutskever, I., Chen, K., Corrado, G. and Dean, J., "Distributed representations of words and phrases and their compositionality," 2013.

Mullainathan, S. and Spiess, J., "Machine learning: An applied econometric approach," Journal of Economic Perspectives, vol. 31, no. 2, May 2017, pp. 87-106.

Ortega-Bastida, J., Gallego, A.-J. and Pertusa, A., "Multimodal object recognition using deep learning representations extracted from images and smartphone sensors," in CIARP, 2019, pp. 521-529.

Pagolu, V. S., Challa, K. N. R., Panda, G. and Majhi, B., "Sentiment analysis of twitter data for predicting stock market movements," 2016.

Richardson, A., van Florenstein Mulder, T. and Vehbi, T., "Nowcasting new zealand gdp using machine learning algorithms," Centre for Applied Macroeconomic Analysis, CAMA Working Papers 2018-47, 2018.

Shi, X., Chen, Z., Wang, H., Yeung, D., Wong, W. and Woo, W., "Convolutional lstm network: A machine learning approach for precipitation nowcasting," in NIPS, 2015, pp. 802-810.

Trask, A., Michalak, P. and Liu, J., "sense2vec - a fast and accurate method for word sense disambiguation in neural word embeddings," 2015.

van der Maaten, L. and Hinton, G., "Visualizing high-dimensional data using t-sne," Journal of Machine Learning Research, vol. 9, no. nov, 2008, pp. 2579-2605.

Vapnik, V., Statistical learning theory. John Wiley and Sons, 1998.

Zukime, M. and Junoh, M., "Predicting gdp growth in malaysia using knowledge-based economy indicators: a comparison between neural network and econometric approaches," Sunway Academic Journal, 2004, pp. 39-50. 